\title{
Mutations in the long QT gene, KCNQ1, are an uncommon cause of atrial fibrillation
}

\author{
P T Ellinor, R K Moore, K K Patton, J N Ruskin, M R Pollak, C A MacRae
}

Heart 2004;90:1487-1488. doi: 10.1136/hrt.2003.027227

A trial fibrillation $(\mathrm{AF})$ is the most common clinical arrhythmia and a major source of morbidity and mortality. ${ }^{1}$ The underlying mechanisms for AF are highly heterogeneous and often related to associated structural heart disease. ${ }^{2}$ However, in several large studies 10-30\% of subjects have no obvious heart disease, and are labelled as primary or lone AF. ${ }^{1}$

The identification of extended families with lone $\mathrm{AF}$, and the mapping of discrete genetic loci in such families, raises the possibility that the heritable predisposition to this form of the arrhythmia is accessible. ${ }^{3}{ }^{4}$ Recently, the first gene for an inherited form of AF was identified in a family with autosomal dominant transmission. ${ }^{5}$ A mutation was found in the first transmembrane spanning domain of the potassium channel gene, KCNQ1, resulting in serine-glycine substitution (S140G). Mutations in other residues throughout this gene cause long QT syndrome type l (LQT1). Coexpression of the S140G mutant allele with KCNEl in cells results in a gain of function with increased current density, and an alteration of channel activation and inactivation. These observations contrast with the reduction in current density seen with LQT1 associated mutations. The unique phenotype associated with this activating mutation includes persistent AF and, surprisingly, QT prolongation. This paradoxical result may reflect chamber specific effects of the mutation, differential imprinting of the gene in atrium and ventricle, or coupling of the channel with different partner proteins, but emphasises that our understanding of repolarisation is incomplete.

The heritable contribution to the majority of lone AF cases is less clear. Given the importance of AF we sought to determine whether mutations in KCNQ1 play any role in more typical forms of this arrhythmia.

\section{METHODS}

Serial subjects with lone AF referred to the arrhythmia service were enrolled between 1 June 2001 and 16 December 2002. Inclusion criteria were ECG documented AF, and an age $\leqslant 65$ years. The exclusion criteria were: structural heart disease on echocardiography, a history of hypertension, rheumatic heart disease, hyperthyroidism, myocardial infarction, or congestive heart failure.

Each subject underwent a physical examination and a standardised interview to identify past medical conditions, medications, symptoms, and possible triggers for initiation of AF. All subjects were evaluated by 12 lead ECG, echocardiogram, and a blood sample for DNA analysis was obtained.

Oligonucleotide primers for polymerase chain reaction (PCR) amplification of the coding region of KCNQ1 (available on request) were designed for exons $1 \mathrm{~A}, 1 \mathrm{~B}$, and 2-15 using the known cDNA (NM 000218) and genomic sequence.

PCR was performed under standard conditions and after determination of the melting profile, amplicons were analysed for mutations using denaturing high performance liquid chromatography (DHPLC). The sensitivity of DHPLC in

\begin{tabular}{|c|c|c|}
\hline Exon & Polymorphism & Allele frequency (\%) \\
\hline 1 & $\begin{array}{l}\text { gg insertion } 3^{\prime}+81 \\
\mathrm{G} 174 \mathrm{~A}, \mathrm{~T} 58 \mathrm{~T}\end{array}$ & $\begin{array}{l}3.9 \\
1.06\end{array}$ \\
\hline $1 \mathrm{~A}$ & $c->+5^{\prime}$ UTR - 129 & 0.7 \\
\hline 2 & C228T, Y76Y & 0.7 \\
\hline 5 & $c^{-}>+3^{\prime}+8$ & 0.35 \\
\hline 10 & $c->+5^{\prime}-14$ & 0.35 \\
\hline 11 & $t->c 3^{\prime}+14$ & 9.6 \\
\hline \multirow[t]{2}{*}{12} & G1353A, S452S & 20.2 \\
\hline & $g->a 3^{\prime}+36$ & 37.6 \\
\hline 13 & $t->c 3^{\prime}+43$ & 22.3 \\
\hline \multirow[t]{2}{*}{15} & G1515A, T505T & 0.35 \\
\hline & C1701T, Y567Y & 22.7 \\
\hline \multicolumn{3}{|c|}{$\begin{array}{l}\text { The polymorphisms are numbered based on the start codon using the } \\
\text { cDNA sequence NM } 000218 \text {. Lower case letters refer to intronic bases } \\
\text { and are located with respect to the corresponding intron-exon } \\
\text { boundaries }\left(5^{\prime} \text { or } 3^{\prime}+/- \text { number of base pairs). Upper case letters refe }\right. \\
\text { to exonic bases. None of the observed polymorphisms changed amino } \\
\text { acid sense. }\end{array}$} \\
\hline
\end{tabular}

the context of the KCNQl gene, estimated by direct sequencing, was over $98 \%$. Samples were initially screened by DHPLC, and positive amplicons were subsequently sequenced using the dye terminator method on an $\mathrm{ABI}$ fluorescent sequencer. Exon 1, which contains the previously reported S140G mutation, was sequenced in all 141 subjects. Statistical analyses were performed using STATA version 8.0.

\section{RESULTS}

One hundred and forty one unrelated study subjects were enrolled. The mean age at onset of AF was 45 years, and the mean age at enrolment was 54 years. All study subjects presented initially with paroxysmal AF, and 15\% have since progressed to persistent $\mathrm{AF}$. The mean resting heart rate and systolic and diastolic blood pressure were normal at enrolment.

The ECGs of the study subjects were notable for a normal mean PR (175.8 ms; 34), QRS (92.0 ms; 12) and QT $(402.8 \mathrm{~ms} ; 46)$ intervals. Eighteen of the study subjects $(12.8 \%)$, of whom 15 were on flecainide, were found to have a QTc of greater than $450 \mathrm{~ms}$. Echocardiography of the study cohort revealed a mean left atrial size at the upper limits of normal (38.8 (6.4) $\mathrm{mm})$ and a normal left ventricular ejection fraction $(0.62(6.6))$.

The coding sequence of KCNQ1 was screened for mutations using a combination of DHPLC and direct sequencing. While 15 polymorphisms were identified, no disease causing mutations were found (table 1). None of these polymorphisms result in changes in protein sequence, and three of the polymorphisms have been described previously.

\section{DISCUSSION}

To date little attention has focused on the relation between $\mathrm{AF}$ and prolongation of the QT interval. Maintenance of AF is 
dependent on vagal stimulation in multiple model systems, and this dependence is thought to reflect the role of dispersion of refractoriness in the sustained propagation of electrical rotors within the atria. ${ }^{2}$ Atrial repolarisation has not been extensively studied in the context of long QT syndrome, but in at least one form of the disorder abnormalities of atrial electrophysiology have been documented. In long QT syndrome type 4 (LQT4), caused by mutations in the $\beta$-ankyrin gene, affected family members exhibit not only typical ventricular repolarisation abnormalities, but also sinoatrial dysfunction and AF. Recently, Chen and colleagues identified an unusual mutation in the LQT1 potassium channel gene, KCNQ1, that resulted in very early onset AF and long QT syndrome. ${ }^{5}$ These findings, coupled with the variable sensitivity of the surface ECG in inherited repolarisation disorders, and the identification of a sizeable proportion of our cohort with prolongation of the QT interval, led us to investigate a role for mutations in the KCNQ1 gene in lone AF probands.

Using DHPLC screening and direct sequencing, we found no evidence of KCNQ1 mutations in 141 unrelated individuals with lone AF. These data effectively exclude KCNQ1 mutations as a common cause of AF, and suggest that those families that do have mutations at this locus are unlikely to exhibit a typical lone AF phenotype. Any QT prolongation observed in our study cohort appears to be related to the use of antiarrhythmic medications rather than an underlying genetic predisposition.

Our findings further support the need for systematic genetic studies. This cohort was derived from referrals to an academic medical centre and is largely of Northern European descent, thus may not be reflective of all cohorts with lone AF. Eventually, the identification of the causative genes at previously described genetic loci will elucidate the pathophysiology of this morbid condition. ${ }^{34}$

\section{ACKNOWLEDGEMENTS}

This project is supported by an NIH award to Dr Ellinor (HL71632).

\section{Authors' affiliations}

M R Pollak, Department of Medicine, Brigham and Women's Hospital and Harvard Medical School, Boston, Massachusetts, USA

P T Ellinor, R K Moore, K K Patton, J N Ruskin, C A MacRae, Cardiovascular Research Center and Cardiac Arrhythmia Service Massachusetts General Hospital, Boston, Massachusetts, USA

Correspondence to: Dr Calum A MacRae, Cardiovascular Research Center, 149 13th Street, 4th Floor Charlestown, MA 02129, USA; cmacrae@partners.org

Accepted 26 February 2004

\section{REFERENCES}

1 Chugh SS, Blackshear JL, Shen WK, et al. Epidemiology and natural history of atrial fibrillation: clinical implications. J Am Coll Cardiol 2001;37:371-8.

2 Nattel S. New ideas about atrial fibrillation 50 years on. Nature 2002;415:219-26.

3 Brugada R, Tapscott T, Czernuszewicz GZ, et al. Identification of a genetic locus for familial atrial fibrillation. N Engl J Med 1997;336:905-11.

4 Ellinor PT, Shin JT, Moore RK, et al. Locus for atrial fibrillation maps to chromosome 6q14-16. Circulation 2003;107:2880-3.

5 Chen $\mathrm{YH}, \mathrm{Xu}$ SJ, Bendahhou S, et al. KCNQ1 gain-of-function mutation in familial atrial fibrillation. Science 2003;299:251-4.

\section{ELECTRONIC PAGES}

\section{Heart Online case reports: www.heartinl.com}

7 he follow electronic only articles are published in conjunction with this issue of Heart. Altered dystrophin expression in the right atrium of a
patient after Fontan procedure with atrial flutter

C J McMahon, M Vatta, C D Fraser Jr, J A Towbin, A C Chang

Underlying mechanisms in the development of atrial flutter or intra-atrial re-entry tachycardia in patients with structural cardiac abnormalities remain poorly defined. The right atrial myocardium from two patients with congenital heart disease was evaluated, of whom one presented with severe right atrial dilation and arrhythmia and the other with a normal right atrium, to assess whether increased right atrial pressure and volume overload give rise to sarcolemmal alteration. $\mathrm{N}$ terminus dystrophin staining in the atrium from the patient who had undergone a Fontan procedure showed a normally distributed but significantly reduced staining signal compared with the second patient. This is the first report that patients with severe right atrial dilation and atrial flutter have marked reduction in atrial dystrophin expression.

(Heart 2004;90:e65) www.heartjnl.com/cgi/content/full/90/ $12 / \mathrm{e} 65$

\footnotetext{
Lipomatous hypertrophy of the interatrial septum: a commonly misdiagnosed mass often leading to unnecessary cardiac surgery

I Nadra, D Dawson, S A Schmitz, P P Punjabi, P Nihoyannopoulos

Lipomatous hypertrophy of the interatrial septum is a benign cardiac mass that should be considered as part of the
}

differential diagnosis for any atrial cardiac tumour. In the reported case, this lesion was initially suspected to be malignant and the patient was thus referred directly to cardiac surgeons for surgical removal. Unnecessary surgical intervention was swiftly averted because the cardiac surgeon promptly referred the patient for an expert echocardiogram that confirmed the diagnosis of lipomatous hypertrophy. The authors discuss the characteristic features of this lesion and how the diagnosis may be made based on several noninvasive imaging modalities without the need for a tissue biopsy. This condition is more common than initially thought and remains under-recognised by most clinicians. In such cases an increased awareness of this lesion along with the opinion of a specialist echocardiologist would help to avoid a misdiagnosis and unnecessary intervention.

(Heart 2004;90:e66) www.heartjnl.com/cgi/content/full/90/ 12/e66

\section{Apical ballooning in relatives}

L Pison, P De Vusser, W Mullens

Apical ballooning of the left ventricle was first introduced as takotsubo-like left ventricular dysfunction in 1990 by Satoh and colleagues. The syndrome is characterised by reversible extensive akinesia of the apical and mid-portions of the left ventricle with hypercontraction of the basal segment. For the first time two sisters with this syndrome are reported, suggesting a possible genetic aetiology.

(Heart 2004;90:e67) www.heartjnl.com/cgi/content/full/90/ $12 / \mathrm{e} 67$ 